\title{
Evaluation of microvessel density with CD31 and CD105 in patients with psoriasis under methotrexate and acitretin therapy
}

\author{
Ebru Zemheri' ${ }^{1}$, Ayşe Serap Karadağ², Ilkin Zindanci ${ }^{3}$, Pınar Engin Zerk ${ }^{4}$, Melike Kibar Ozturk ${ }^{3}$ \\ 1Pathology Clinic, İstanbul Ümraniye Training and Research Hospital, University of Health Sciences, Istanbul, Turkey \\ 2Dermatology Clinic, Istanbul Medeniyet University, School of Medicine, Goztepe Research and Training Hospital, Istanbul, Turkey \\ ${ }^{3}$ Dermatology Clinic, İstanbul Ümraniye Training and Research Hospital, University of Health Sciences, Istanbul, Turkey \\ ${ }^{4}$ Pathology Clinic, Okmeydani Training and Research Hospital, Istanbul, Turkey
}

Adv Dermatol Allergol 2020; XXXVII (3): 422-427 DOI: https://doi.org/10.5114/ada.2019.87279

\begin{abstract}
Introduction: Methotrexate and acitretin are known to be effective in the treatment of psoriasis, but the mechanisms of the effects of these drugs are not fully known.

Aim: To investigate the effect of methotrexate and acitretin on microvessel density (MVD) in psoriasis.

Material and methods: Eighteen patients with psoriasis treated with methotrexate and 9 patients with psoriasis treated with acitretin (AT) were included in this study. MVD was evaluated immunohistochemically by using CD31 and CD105 (endoglin) antibodies.

Results: In the methotrexate group, the decrease in CD31 levels after treatment was found to be statistically significant, while in the AT group it was found to be highly significant. In both methotrexate and AT group, there was a statistically highly significant decrease in CD105 levels after treatment. There was no statistically significant difference between CD31 measurements of methotrexate and AT groups. When CD105 levels were measured before and after treatment, no statistically significant difference was found between methotrexate and AT. According to the results of CD31 changes before and after treatment, the CD31 difference was not statistically significant in both groups while the difference was higher in the AT group. CD105 differences were not statistically significant in both treatment groups before and after treatment.

Conclusions: CD31 and CD105 dyes indicate the effects of therapies on vascular proliferation and may be indicators that can be used in daily routine and follow-up studies for psoriasis.
\end{abstract}

Key words: methotrexate, acitretin, microvessel density, CD31, CD105.

\section{Introduction}

Psoriasis is a papulosquamous disease with a prevalence of $1-2 \%$. It is characterized clinically by recurrent erythematous squamous plaques. Histologically parakeratosis, regular acanthosis, granular layer loss, Munro and Kogoj abscesses as well as vascular changes are observed [1]. These vascular alterations are in the form of increased and dilated capillary vessels and increased twisted immature veins with elevated permeability [2]. Angiogenic activity in psoriasis has been reported to be associated with cell-cell interaction, cytokine-mediated humoral response, development and growth factors, matrix metalloproteinase release, blood coagulation and fibrinolysis [3].
Endoglin (CD105) is a transforming growth factor receptor and regulates TGF signalling. It is particularly expressed in vascular vessels that accompany inflammatory and neoplastic situations. It shows a stable expression in normal vascular structures while displaying a high expression in increased angiogenic activity [3, 4]. Endoglin is proposed as a vascular marker in patients with angiogenic disease or with a solid tumour for imaging tumour in the prognosis and therapeutic monitoring [5]. In a study that explores tumour angiogenesis in colon carcinomas, endoglin was found to be more sensitive and specific compared to the other indicators. For this reason, CD31 is regarded as a more practical marker than CD34 and factor VIII in assessing the increase in vascu-

Address for correspondence: Assoc. Prof. Ebru Zemheri, Pathology Clinic, İstanbul Ümraniye Training and Research Hospital, University of Health Sciences, 34760 Umraniye, Istanbul, Turkey, phone: +90 21663218 18, e-mail: ebruzemheri@gmail.com Received: 2.11.2018, accepted: 19.11.2018. 
larity [6]. Van De Kerkhof et al. had shown an increase of endoglin expression in psoriatic plaque and a decrease in endoglin expression in the healthy zones [4]. Endoglin was also identified as a proangiogenic molecule in the blood of patients with psoriasis [3].

Platelet endothelial cell adhesion molecule-1 (PECAM-1) is a member of the family of cell adhesion molecules: CD31. It is expressed in natural killer cells, mast cells and $T$ and $B$ lymphoid cells, on the lateral face of endothelin and on the surface of the neutrophil, monocyte, and platelet. CD31 plays a role in angiogenesis, platelet collection, homeostasis, and in barrier function of the vascular endothelium. Its expression is increased in newly formed blood and lymphatic vessels [7].

Synthetic retinoid acitretin (AT) and folic acid analogue methotrexate are the most commonly used drugs in the treatment of psoriasis. Although studies on the mechanism of action of both drugs in psoriasis have been carried out, their mechanisms of action have not been fully elucidated and new findings are obtained that contribute to pathogenesis every day [8].

\section{Aim}

The aim of this study was to investigate the effect of methotrexate and AT treatment on angiogenesis in psoriasis and to find out if there is a relationship or difference between CD31 and CD105 when evaluating angiogenesis.

\section{Material and methods}

Eighteen patients under methotrexate and ten patients under AT treatment were included in the study. 18 of the patients were females and 10 of them were males. The patients took methotrexate therapy orally at 10-15 mg/week and AT 0.30-0.50 mg/kg/day. All topical and systemic treatments were stopped 2 weeks and 2 months, respectively, prior to the onset of the study. A $4 \mathrm{~mm}$ diameter punch biopsy specimen was obtained from the plaque-type lesion in the trunk of all patients before and after treatment. Formalin fixed tissues were examined after routine laboratory procedures. In the immunohistochemical analysis, CD105 (Dako, Rabbit PAb RB-9097-R1 53, Fremont, CA, USA) and CD31/PECAM-1 (endothelial cell marker) (Ab-1 Diagnostic Biosystems Clone JC/70A) were used. Dyeing was performed on Bond Autostainer. Microvessel density (MVD) was evaluated with CD31 and CD105. The evaluation was performed by counting the veins in the papillary and subpapillary area at 40× magnification in the light microscope. The endothelial cells that were single or made groups were counted as a single vessel with or without lumens.

\section{Statistical analysis}

For statistical analysis, NCSS (Number Cruncher Statistical System) 2007 \& PASS (Power Analysis and Sample
Size) and 2008 Statistical Software (Utah, USA) programs were used. In evaluating study data, descriptive statistical methods (mean, standard deviation, median) were used. The Mann Whitney $U$ test was used for the comparison of variables between groups and Wilcoxon sign test was used for pre- and post-treatment comparisons. Significance was evaluated at $p<0.05$.

\section{Results}

In the Methotrexate group, the decrease in CD31 levels after treatment was found to be statistically significant ( $p=0.031 ; p<0.05)$. The CD31 levels in the AT group were found to be highly significant $(p=0.005 ; p<0.01$ ). In both methotrexate and AT group, there was a statistically significant decrease in CD105 levels after treatment ( $p=0.001$ and $p=0.005$, respectively). There was no statistically significant difference between CD31 measurements of methotrexate and AT groups ( $p=0.314$; $p=0.581 ; p>0.05)$. When CD105 levels were measured before and after treatment, no statistically significant difference was found between Methotrexate and AT groups ( $p=0.502 ; p=0.249 ; p>0.05)$. The CD31 differences before and after treatment were not statistically significant in both groups while the difference was higher in AT group ( $p=0.250, p>0.05)$. CD105 differences were not statistically significant in both groups before and after treatment ( $p=962, p>0.05)$ (Figures 1 and 2).

\section{Discussion}

Psoriasis is a disease induced by Th1 and Th17 cells and it is known that aberrant keratinocyte and endothelial proliferation contributes to the disease. The size of the vascular structures observed in the psoriatic lesion is larger than the normal skin. Many studies have shown that these vessels are wide, dilated and curved. This change is more pronounced in the papillary dermis. The increase in histologically observed vascular structures has been found to be related to vascular endothelial growth factor, angiopoietin and proangiogenic cytokines such as TNF, IL-8 and IL-7 $[9,10]$. In addition, it has been found that adhesion molecules that induce leukocyte aggregation such as ICAM-1, VCAM-1, Thy-1, E- and P-selectin are also released from these vascular structures [11].

New technological and experimental studies investigating the pathogenesis of psoriasis due to these prominent vascular changes focus on angiogenesis. It is important to target the vascular structure in the psoriatic lesions, even in treatment methods that do not specifically affect angiogenesis. It is not clear whether this interaction is the result of treatment or as a result of remission of the lesion; however, it is known that the vascular component of the disease is closely related to the changes observed in clinical symptoms. Therefore, angiogenesis is considered a potential target for specific 

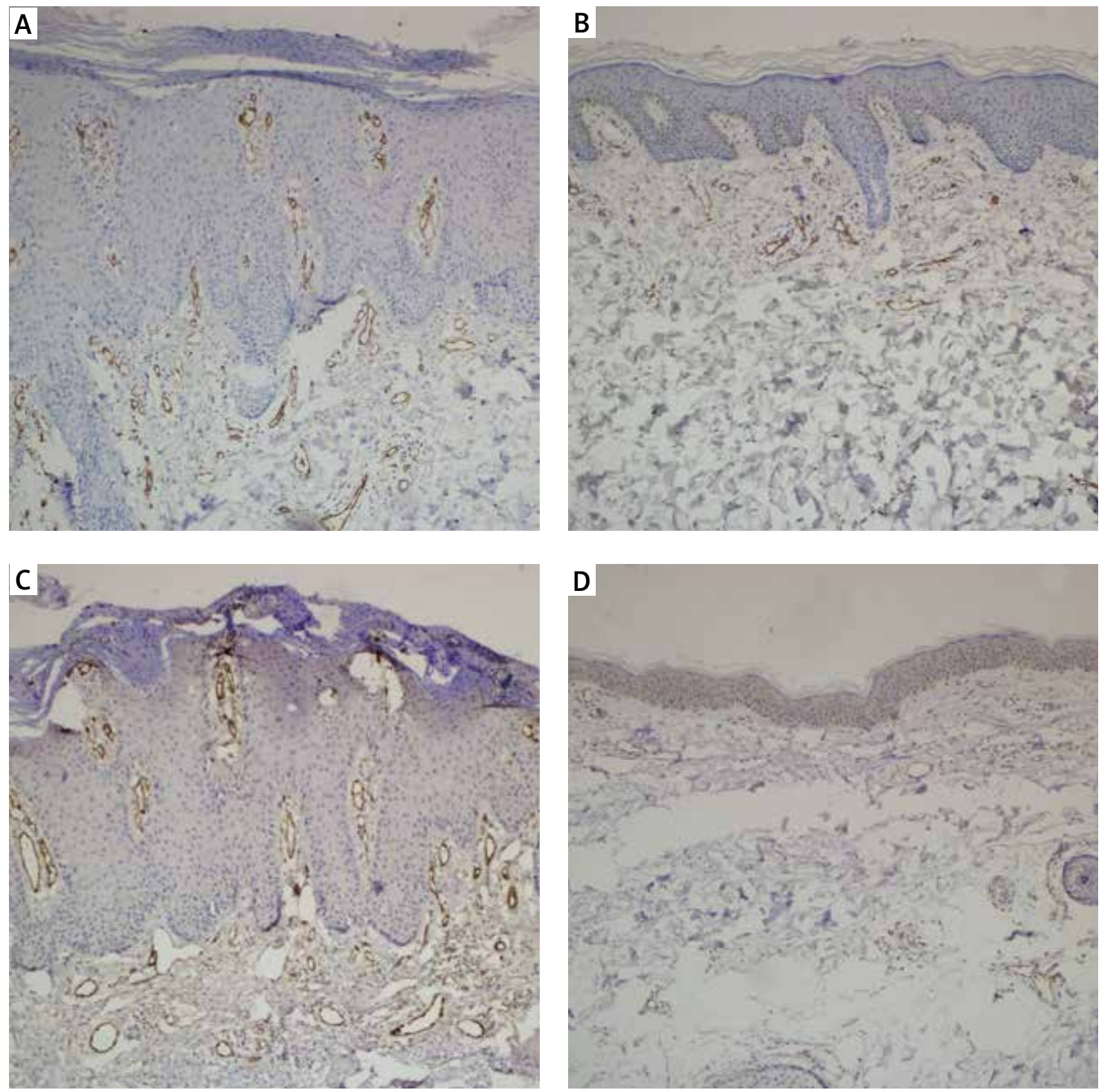

Figure 1. A - Microvessel density evaluated with CD31 before treatment with acitretin. B - Microvessel density evaluated with CD31 after treatment with acitretin. C - Microvessel density evaluated with CD105 before treatment with acitretin. D - Microvessel density evaluated with CD105 after treatment with acitretin

therapies for psoriasis. The results of antiangiogenic agents in clinical trials are limited. However, promising results are obtained in animal experiments [11].

Methotrexate is a folic acid analogue and it is an antiproliferative agent. Although the mechanism of action in psoriasis is not known, it has been shown to inhibit the adhesion molecules released by both $T$ lymphocytes and endothelial cells and thus prevent the collection of leukocytes $[8,12,13]$. AT is a synthetic retinoid which is widely used in the treatment of psoriasis. Its role in the treatment is not well understood and there are reports showing that it reduces keratinocytes proliferation, inhibits inflammation and suppresses angiogenesis. Its effect on angiogenesis is thought to be both direct and indirect by reducing endothelial migration and suppressing VEGF production $[12,14,15]$.

There are a limited number of histopathological investigations reporting the morphological changes before and after treatment. Eskicirak et al. evaluated both clinical improvement and histopathological changes after $1 \%$ topical methotrexate treatment. There was a $20 \%$ decrease in papillary dermal capillaries and his- 

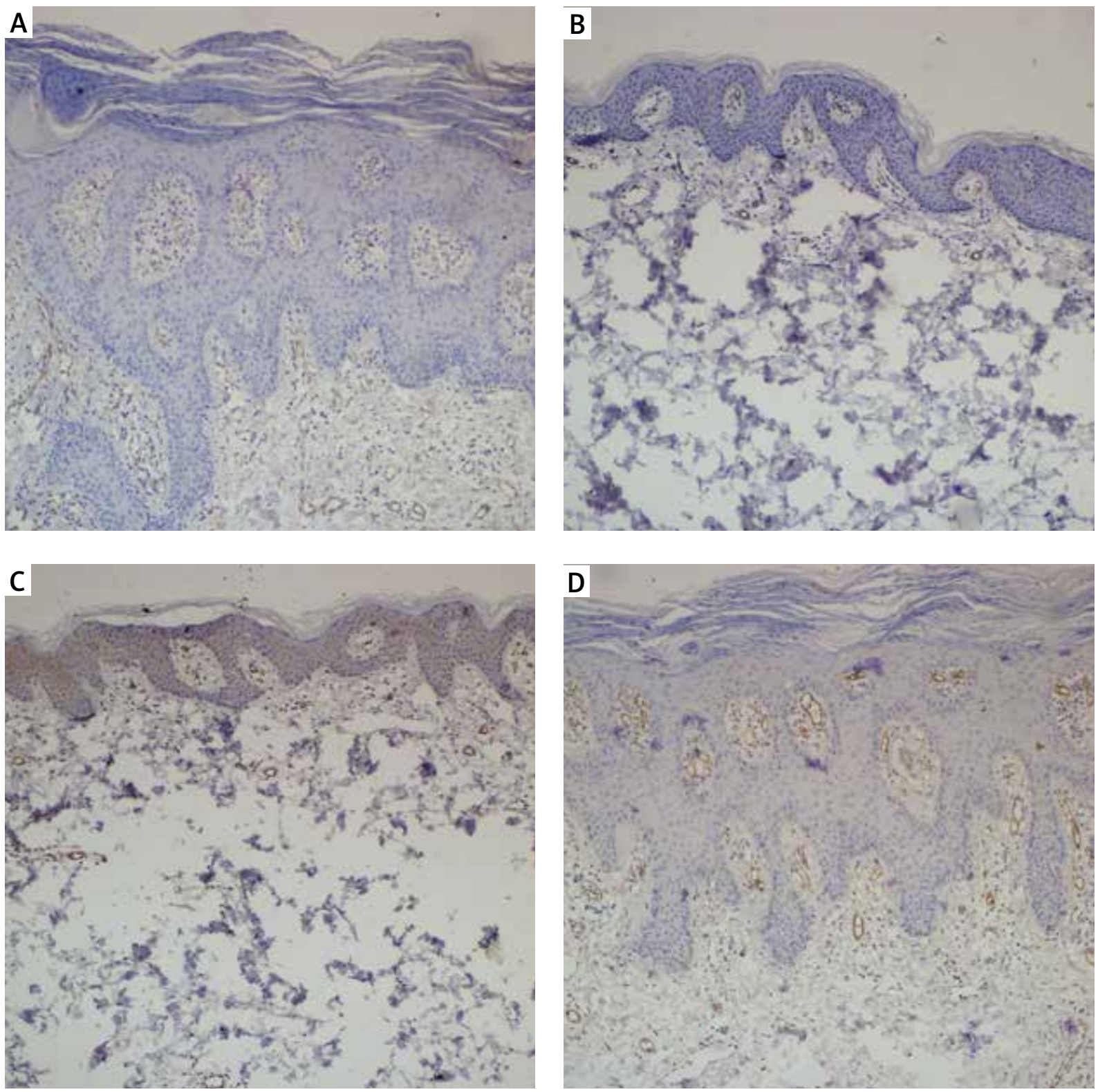

Figure 2. A - Microvessel density evaluated with CD31 before treatment with methotrexate. B - Microvessel density evaluated with CD31 after treatment with methotrexate. C - Microvessel density evaluated with CD105 before treatment with methotrexate. D - Microvessel density evaluated with CD105 after treatment with methotrexate

topathological improvement was statistically significant [16]. In another morphological study, histopathological findings were evaluated before and after phototherapy, AT and methotrexate treatment. There was a decrease in dilated vascular structures after treatment with both AT and methotrexate but this was not statistically significant [17].

Yamasaki et al. investigated the efficacy of low-dose methotrexate in psoriasis. They reported that methotrexate inhibited neovascularization as well as suppression of TNF- $\alpha$ induced by ICAM-1 and VCAM-1 released from vascular endothelial cells [18]. In another study, CD31 and ICAM staining were performed for clinical, histopathological and vascular changes before and after methotrexate treatment. In addition to significant reduction in inflammation with epidermal hyperplasia after treatment, CD31, ICAM and vascularization were also decreased. As a result, it was concluded that methotrexate reduces revascularization [8]. In another study comparing methotrexate and PUVA therapy in patients with psoriasis, methotrexate was found to be a more potent antiangiogenic factor, leading to a more pronounced re- 
duction in vascularity $[13,19]$. Methotrexate is an agent used in many diseases as well as psoriasis, and it has been reported to reduce angiogenesis in the cornea and nasal polyps [20, 21].

There are few publications evaluating vascular changes after acitretin use but there are no publications evaluating vascular structures immunohistochemically. In a study investigating the changes observed in vascular structures in videocapillaroscopy, a 65.4\% reduction in vascular structures after AT therapy was reported [15]. Kim et al. investigated VEGF expression in the epithelium, dermis and vascular structures in their study on the effect of AT on VEGF and found that AT reduces angiogenesis by suppressing VEGF [9].

In this study which evaluated vascular changes after methotrexate and AT treatment, we observed a significant decrease in vascularity with both CD31 and endoglin after treatment in both groups. This decline was at a higher level in the EC group. There are similar studies with methotrexate whose results are parallel to the findings in this study [8]. There are publications evaluating the effectiveness of AT on vascular structures with videocapillaroscopy and VEGF expression [9, 15]. However, there exists no study in the literature showing changes in the vascular structures with markers such as CD31 or endoglin. In this respect, the present study is the first one focusing on immunohistochemical changes.

We observed changes in vascular structures with two different markers. We utilized endoglin which is shown to be more effective in the evaluation of vascular changes [6] and the commonly used CD31; we did not observe any difference in both groups. Therefore, we think that these markers can be used routinely in laboratories for evaluation of vascular changes in psoriasis. Our study had several limitations, including a relatively small number of participants and lack of comparative histopathological and immunohistochemical evaluations with other conservative treatment options.

\section{Conclusions}

Conservative therapies which are widely used in psoriasis like methotrexate and AT are effective in reducing the vascular proliferation. CD31 and CD105 dyes indicate the effects of therapies on vascular proliferation and may be indicators that can be used in daily routine and followup studies.

\section{Conflict of interest}

The authors declare no conflict of interest.

\section{References}

1. Griffiths CE, Barker JN. Pathogenesis and clinical features of psoriasis. Lancet 2007; 370: 263-71.
2. Barton SP, Abdullah MS, Marks R. Quantification of microvascular changes in the skin in patients with psoriasis. Br J Dermatol 1992; 126: 569-74.

3. Pohl D, Andrýs C, Borská L, et al. Serum Level of a soluble form of endoglin (CD105) is decreased after Goeckerman's therapy of psoriasis. Acta Med 2011; 54: 59-62.

4. Van De Kerkhof PCM, Rulo HFC, Van Pelt JPA, et al. Expression of endoglin in the transition between psoriatic uninvolved and involved skin. Acta Derm Venereol 1998; 78: 19-21.

5. Duff SE, Li C, Garland JM, Kumar S. CD105 is important for angiogenesis: evidence and potential applications. FASEB J 2003; 17: 982-94.

6. Saad RS, Liu YL, Nathan G, et al. Endoglin (CD105) and vascular endothelial growth factor as prognostic markers in colorectal cancer. Modern Pathol 2004; 17: 197-203.

7. Tursen B, Kara T, Tursen $U$, et al. The changes in expression of Ki-67, and CD31 in psoriatic lesions before and after etanercept treatment. Hong Kong I Dermatol Venereol 2013; 21: 5-13.

8. Yazici AC, Tursen U, ApaDD, et al. The changes in expression of ICAM-3, Ki-67, PCNA, and CD31 in psoriatic lesions before and after methotrexate treatment. Arch Dermatol Res 2005; 297: 249-55.

9. Kim CY, Kim SM, Kim GD. The effect of acitretin to the expression of vascular endothelial growth factor in psoriasis. J Life Sci 2009; 19: 327-33.

10. Heidenreich R, Rocken M, Ghoreschi K. Angiogenesis drives psoriasis pathogenesis. Int J Exp Path 2009; 90: 232-48.

11. Guérard S, Pouliot R. The role of angiogenesis in the pathogenesis of psoriasis: mechanisms and clinical implications. J Clin Exp Dermatol Res 2012; S: 2.

12. Martins GA, Arruda L. Systemic treatment of psoriasis part I: methotrexate and acitretin. An Bras Dermatol 2004; 79: 263-78.

13. Shaker OG, Khairallah M, Abdel-Halim MR, Abuzeid OM. Antiangiogenic effect of methotrexate and PUVA on psoriasis. Cell Biochem Biophys 2013; 67: 735-42.

14. Nofal A, Al-Makhzangy I, Attwa E, et al. Vascular endothelial growth factor in psoriasis: an indicator of disease severity and control. J Eur Acad Dermatol Venereol 2009; 23: 803-6.

15. Stinco G, Buligan C, Errichetti E. Clinical and capillaroscopic modifications of the psoriatic plaque during therapy: observations with oral acitretin. Dermatol Res Pract 2013; 2013: 781942.

16. Eskicirak B, Zemheri E, Cerkezoglu A. The treatment of psoriasis vulgaris: $1 \%$ topical methotrexate gel. Int J Dermatol 2006; 45: 965-9.

17. Ozkanli S, Zemheri E, Karadag AS, et al. A comparative study of histopathological findings in skin biopsies from patients with psoriasis before and after treatment with acitretin, methotrexate, and phototherapy. Cutan Ocul Toxicol 2015: 34: 276-81.

18. Yamasaki E, Soma Y, Kawa Y, Mizoguchi M. Cutaneous biology methotrexate inhibits proliferation and regulation of the expression of intercellular adhesion molecule- 1 and vascular cell adhesion molecule-1 by cultured human umbilical vein endothelial cells. Br J Dermatol 2003; 149: 30-8.

19. Park SK, Kim HI, Yang YI, Hur DY. Effects of methotrexate on vascular endothelial growth factor, angiopoietin 1 , and angiopoietin 2 in nasal polyps. Am J Rhinol Allergy 2011; 25: e129-32. 
20. Mohamed O, Abu-Zeid M, Hussin Rashid HM, El-Said Abdel-Haleem MR. Effect of methotrexate and puva on the amount of vascular endothelial growth factor expression and capillary perfusion of psoriatic lesions. Olfat Gamil Shaker Cairo University Giza, Egypt Doctorial (PhD) Thesis 2007.

21. Joussen AM, Kruse FE, Völcker HE, Kirchhof B. Topical application of methotrexate for inhibition of corneal angiogenesis. Graefes Arch Clin Exp Ophthalmol 1999; 237: 920-7. 